\title{
Clinical impact of cerebral microbleeds on cognition in patients with CADASIL
}

\author{
Jung Seok Lee ${ }^{1}$, Keun Hyuk $\mathrm{Ko}^{2}$, Jung-Hwan $\mathrm{Oh}^{1}$, Jay Chol Choi ${ }^{1}$, Joong-Goo Kim ${ }^{2, *}$ \\ ${ }^{1}$ Department of Neurology, Jeju National University College of Medicine \\ ${ }^{2}$ Department of Neurology, Jeju National University Hospital
}

\begin{abstract}
Cerebral autosomal-dominant arteriopathy with subcortical infarcts and leukoencephalopathy (CADASIL) is inherited microangiopathy caused by mutations in the Notch3 gene. Typical findings from brain magnetic resonance imaging (MRI) include subcortical lacunes, extensive white matter change and cerebral microbleeds (CMBs). CMBs are indicative of bleeding-prone microangiopathy. Despite some studies investigating the association between lacunes and cognitive dysfunction in CADASIL, few studies have examined the relationship between cognitive dysfunction and CMBs. We sought to assess whether CMBs are associated with cognitive dysfunction in CADASIL. This study enrolled 83 consecutive patients with CADASIL between April 2012 and January 2014. Their degree of cognitive dysfunction was assessed by the Korean version of the CERAD neuropsychological assessment battery, digit span test, and the Stroop test. A 3.0-T MRI was used to obtain T1-weighted, fluid-attenuated inversion recovery, and susceptibility weighted images. In multiple logistic regression analysis, the grade of CMBs influenced tests of memory dysfunction ( $p=0.003)$. Three or more lacunes correlated with dysfunction in the executive domain $(p=0.013)$ and attention domain $(p=0.005)$. White matter hyperintensity (WMH) was an independent predictor of executive dysfunction ( $p=0.001)$. These findings suggest that in addition to lacunes, $\mathrm{CMBs}$ and WMHs may be useful imaging markers to associated with cognitive dysfunction in CADASIL.
\end{abstract}

Key words: Cerebral autosomal-dominant arteriopathy with subcortical infarcts and leukoencephalopathy (CADASIL), Hypertension, Lacunar infarct, Cognition

\section{INTRODUCTION}

Cerebral microbleeds (CMBs) are the perivascular collections of hemosiderin deposits caused by tiny extravasations of blood. ${ }^{1,2)}$ They can easily be detected by Magnetic Resonance Imaging (MRI) due to recent advances in MRI technology. ${ }^{1,2)}$ Susceptibility-weighted images (SWIs) are high-resolution 3D T2 sequences uniquely attenuated to the detection of hemorrhage. ${ }^{3)}$ The SWI method and smaller section thicknesses are known to be associated

Received: October 23, 2018; Revised: December 2, 2018; Accepted: December 12, 2018

*Correspondence to : Joong-Goo Kim

Department of Neurology, Jeju National University Hospital, 13 Aran-gil,

Jeju-si, Jeju-do 63241, Korea

Tel: 82-64-717-1620, FAX: 82-64-717-1630

E-mail: lilis1118@naver.com with higher rates of CMB detection, especially CMBs on the lobar lesion. ${ }^{4}$ CMBs are frequently detected in patients with ischemic stroke as well as those with intracranial hemorrhage (ICH) in cerebral small vessel disease, which suggests that patients with cerebral small vessel disease have ischemia and CMBs comorbidities. ${ }^{5)}$ Also, previous reports have shown that the presence of CMBs predicts the recurrence of ischemic stroke. ${ }^{6}$ )

Cerebral autosomal-dominant arteriopathy with subcortical infarcts and leukoencephalopathy (CADASIL) is a well known inherited cerebral small vessel disease caused by mutations in the Notch3 gene. ${ }^{7)}$ The main clinical manifestations are a recurrent stroke, cognitive decline, chronic headache, mood disturbances, and seizure ${ }^{8,9)}$ Differences between Caucasian and Asian CADASIL patients concerning neuroimaging features include higher rates of 
ICH in East Asians. ${ }^{10)} \mathrm{ICH}$ has rarely been described in Caucasian patients with CADASIL. ${ }^{11)}$ Despite some studies investigating the association between lacunes and cognitive dysfunction in CADASIL, few studies have examined the relationship between cognitive dysfunction and CMBs. We are aware of only one prior study that found a longitudinal association between CMBs and cognition. ${ }^{12)}$ Therefore, we sought to assess whether CMBs are associ-

Table 1. Demographic characteristics $(n=83)$

\begin{tabular}{lc}
\hline Age, year & $62.5 \pm 12.5$ \\
Male sex & $48(57.8 \%)$ \\
Education, year & $9.5 \pm 5.4$ \\
Hypertension & $49(59.0 \%)$ \\
Diabetes Mellitus & $12(14.5 \%)$ \\
Hypercholesterolemia & $19(22.9 \%)$ \\
Ever-smoking & $34(41.0 \%)$ \\
Number of lacunes, median (range) & $3.0(0-22)$ \\
Number of CMBs, median (range) & $3.0(0-120)$ \\
Scheltens scores & $28.4 \pm 13.6$ \\
\hline
\end{tabular}

$\mathrm{CMBs}=$ cerebral microbleeds. Values are mean \pm SD.
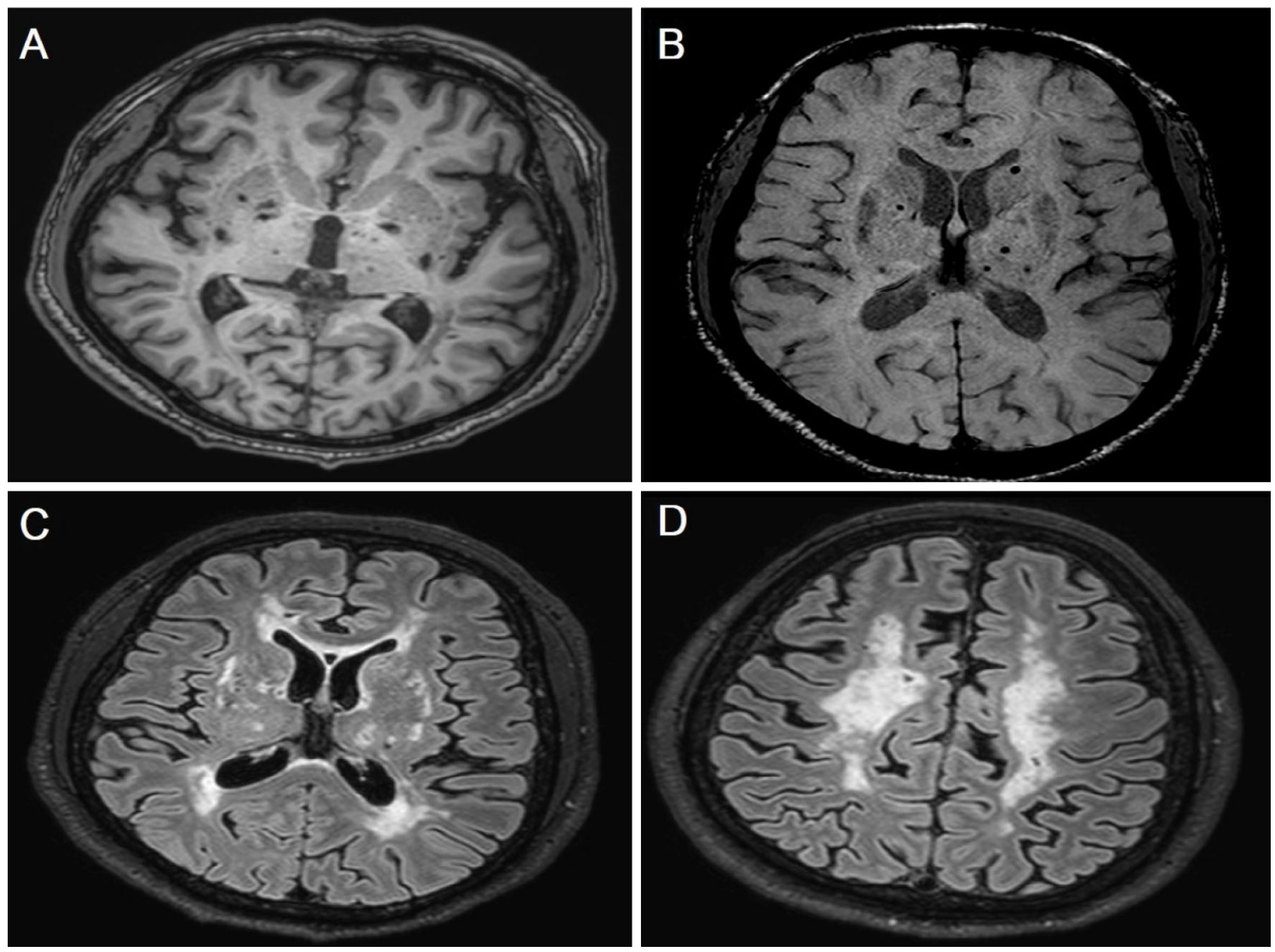

Figure 1. MRI from a 64-year-old patient with CADASIL. (A) 3D T1-weighted image shows lacunes in both basal ganglia. (B) Susceptibility-weighted image shows numerous cerebral microbleeds in both thalami, left the caudate nucleus and right internal capsule. (C-D) Fluid-attenuated inversion recovery image shows periventricular and deep white matter hyperintensities and subcortical lacunes. ated with cognitive dysfunction in patients with CADASIL.

The subjects for this study include consecutive patients who had been diagnosed with CADASIL by genetic testing or skin biopsy analysis at Jeju National University Hospital's Neurology Department between April 2012 and January 2014. We enrolled 86 patients with CADASIL. Patients who did not undergo a MRI examination prospectively $(\mathrm{n}=2)$ or a comprehensive neuropsychological evaluation $(n=1)$ were excluded. Therefore, finally, 83 patients were enrolled.

Data for our analyses were derived from previously reported our study. The details of the study method for this article have been described in detail elsewhere. ${ }^{13)}$

All statistical analyses were performed using SPSS 21.0 (IBM, Armonk, NY, USA). CMBs were classified as ab-

\section{MATERIALS AND METHODS}


Table 2. Univariate logistic analysis of the effects of MRI markers on the dysfunction of each cognitive domain

\begin{tabular}{|c|c|c|c|c|}
\hline Domain & MRI markers & OR & $95 \% \mathrm{CI}$ & $p$ \\
\hline \multirow{9}{*}{ Attention } & CMBs & 1 & & \\
\hline & CMBs (1 2) & 2.00 & $0.35 \sim 11.58$ & 0.439 \\
\hline & CMBs $(\geq 3)$ & 7.33 & $1.91 \sim 28.10$ & 0.004 \\
\hline & Lacunes & 1 & & \\
\hline & Lacunes (1～2) & 4.39 & $0.41 \sim 46.93$ & 0.22 \\
\hline & Lacunes $(\geq 3)$ & 19.83 & $2.45 \sim 160.39$ & 0.005 \\
\hline & Scheltens score & 1.04 & $1.01 \sim 1.08$ & 0.024 \\
\hline & Hypertension & 2.89 & $1.06 \sim 7.91$ & 0.038 \\
\hline & Diabetes Mellitus & 0.61 & $0.15 \sim 2.47$ & 0.492 \\
\hline \multirow{9}{*}{ Executive Function } & CMBs & 1 & & \\
\hline & CMBs $(1 \sim 2)$ & 1.43 & $0.35 \sim 5.79$ & 0.617 \\
\hline & CMBs $(\geq 3)$ & 6.13 & $2.07 \sim 18.18$ & 0.001 \\
\hline & Lacunes & 1 & & \\
\hline & Lacunes (1 2) & 2.4 & $0.54 \sim 10.67$ & 0.250 \\
\hline & Lacunes $(\geq 3)$ & 9.43 & $2.67 \sim 33.29$ & $<0.001$ \\
\hline & Scheltens score & 1.09 & $1.04 \sim 1.14$ & $<0.001$ \\
\hline & Hypertension & 3.16 & $1.27 \sim 7.86$ & 0.0013 \\
\hline & Diabetes Mellitus & 2.06 & $0.57 \sim 7.43$ & 0.272 \\
\hline \multirow{7}{*}{ Memory } & CMBs & 1 & & \\
\hline & CMBs $(1 \sim 2)$ & 7.33 & $1.48 \sim 36.34$ & 0.015 \\
\hline & CMBs $(\geq 3)$ & 10.59 & $2.75 \sim 40.77$ & 0.001 \\
\hline & Lacunes & 1 & & \\
\hline & Lacunes (1 2) & 1.36 & $0.32 \sim 5.89$ & 0.678 \\
\hline & Lacunes $(\geq 3)$ & 3.71 & $1.16 \sim 11.90$ & 0.027 \\
\hline & Scheltens score & 1.05 & $1.02 \sim 1.09$ & 0.005 \\
\hline
\end{tabular}

sent (grade 1), mild (grade 2, the total number of CMBs, 1-2), and moderate to severe (grade $3, \geq 3 \mathrm{CMBs}$ ) according to a grading scale described previously ${ }^{14)}$ because the number of CMBs was not distributed normally. For the same reason, the grade of the lacunes were also grouped as absent (grade 1), mild (grade 2, total number of lacunes, $1-2$ ), and moderate to severe (grade $3, \geq 3$ lacunes), When a variable showed $p<0.20$ in univariate analysis, multiple logistic regression was performed to evaluate the impact of CMBs on scores for the different cognitive domains. The odds ratio for cognitive dysfunction was calculated using a logistic regression analysis that included age, sex, educational level, hypertension, diabetes mellitus, grade of CMBs, grade of lacunes, and degree of white matter hyperintensity (WMH) (semi-quantitative Scheltens scores). The results of the multivariable analysis included only those variables with a $p$-value less than 0.05 . A two-tailed $p$-value $<0.05$ was considered statistically significant.

\section{RESULTS}

Details of the demographics and MRI findings of the subjects with CADASIL are presented in Table 1. The diagnosis was confirmed by NOTCH3 mutation $(n=75)$, and by the skin, a biopsy is showing the presence of granular osmiophilic material $(n=8)$. The sites of mutation were $\operatorname{R544C~}(n=70), \operatorname{R578C}(n=2), \operatorname{R75P}(n=2)$, and C452A $(n=1)$. Sixty-nine subjects were symptomatic, and 14 were asymptomatic. Cerebral infarction was the most frequent manifestation $(n=32$, Fig. 1), followed by chronic headache $(\mathrm{n}=14)$, cognitive dysfunction $(\mathrm{n}=$ $8)$, ICH $(n=8)$, TIA $(n=5)$, and seizure $(n=2)$. All subjects except one had white matter hyperintensity lesions on the FLAIR images. One or more CMBs on SWI were observed in 58 subjects $(69.9 \%)$, and one or more lacunes were seen in $63(75.9 \%$ ) (Fig. 1).

The results of the univariate analysis to evaluate the impact of MRI markers on each cognitive domain are shown in Table 2. All the MRI markers (grade of CMBs, grade of lacunes, and Scheltens scores) were associated with the attention, executive functioning, and memory domains.

In contrast, only the Scheltens scores showed a significant correlation with the language domain. Hypertension was observed in association with the attention and executive functioning, but these associations disappeared in the 
Table 3. Multivariate analyses of the effects of MRI markers on the dysfunction of each cognitive domains

\begin{tabular}{|c|c|c|c|c|c|}
\hline \multirow{2}{*}{ MRI markers } & \multirow{2}{*}{ Domain } & \multirow{2}{*}{ OR } & \multirow{2}{*}{$p$} & \multicolumn{2}{|c|}{$95 \% \mathrm{CI}$} \\
\hline & & & & Lower & Upper \\
\hline $\mathrm{CMBs}(0)$ & memory & & $0.003 * *$ & & \\
\hline CMBs $(1 \sim 2)$ & memory & 7.33 & $0.015^{*}$ & 1.48 & 36.24 \\
\hline CMBs $(\geq 3)$ & memory & 10.59 & $0.001 * *$ & 2.75 & 40.77 \\
\hline Lacunes $(0)$ & attention & & $0.004 * *$ & & \\
\hline Lacunes (1 2) & attention & 4.39 & 0.846 & 0.41 & 46.93 \\
\hline Lacunes $(\geq 3)$ & attention & 19.83 & $0.005 * *$ & 2.45 & 160.39 \\
\hline Lacunes $(0)$ & executive function & & $0.005 * *$ & & \\
\hline Lacunes (1 2) & executive function & 0.84 & 0.846 & 0.14 & 4.91 \\
\hline Lacunes $(\geq 3)$ & executive function & 6.02 & $0.013^{*}$ & 1.47 & 24.68 \\
\hline Scheltens scores & executive function & 1.10 & $0.001 * *$ & 1.04 & 1.16 \\
\hline
\end{tabular}

Adjusted for age, sex, and education level. CMBs $=$ cerebral microbleeds.

$\mathrm{OR}=$ odds ratio; $\mathrm{CI}=$ confidence interval. ${ }^{*} p<0.05, * * p<0.01, * * * p<0.001$

final multivariate model. In the final multivariate logistic regression model (Table 3), the grade of CMBs was independently associated with the memory domain $(p=0.003)$. The moderate to the severe grade of lacunes related to poor performance in the executive domain $(p=0.013)$ and attention domain $(p=0.005)$. Scheltens scores were associated with poor performance in the executive functioning $(p=0.001)$.

\section{DISCUSSION}

Our main finding is that in addition to lacunes, CMBs were associated with memory dysfunction in CADASIL. We also found that WMHs were an independent predictor of executive dysfunction. To our knowledge, this is the first study to detect CMBs using SWI, which is associated with much higher rates of $\mathrm{CMB}$ detection compared with conventional gradient-echo (GRE).

The frequency of CMBs was very common in patients with CADASIL. It ranges from 25 to $69 \%$ in the literature. ${ }^{11,15-17)}$ However, studies on the relationship between CMBs and cognition are very limited in CADASIL patients. One longitudinal study of CADASIL showed an association between a number of CMBs and memory domain, ${ }^{12)}$ whereas other studies did not. ${ }^{18-20)}$ Our results are consistent with the previous longitudinal study showing an association between the number of CMBs and cognitive function, including memory. ${ }^{12)}$ This longitudinal study, however, recruited a small number of patients $(\mathrm{n}=25)$ and did not draw a definite conclusion. ${ }^{20)}$

Our new data conflict with our previous data which did not show an association between CMBs and memory dysfunction.This discrepancy may be explained in part by the number of patients enrollment $(n=83$, new data; $n=40$, previous data) and different methods of assessment. We used more advanced MRI techniques to detect CMBs compared to our previous studies, including (i) the effects of sequences (traditional GRE versus SWI); (ii) section thickness ( $5 \mathrm{~mm}$ versus $2 \mathrm{~mm}$ ); and (iii) field strength (1.5T versus $3 \mathrm{~T}$ ). Additionally, the present study used CERAD$\mathrm{K}$, whereas the previous studies used ADAS-cog K. Unlike, in ADAS- $\operatorname{cog} \mathrm{K}$, the scores could be z-score transformed in CERAD-K.

Our data cannot rule out the possibility that the number of CMBs might not correlate with memory dysfunction in the early stages of CADASIL. However, it should be noted that memory dysfunction is one of the common problems in CADASIL. ${ }^{21,22)}$ Seo et al. reported that the number of CMBs was an independent predictor of multiple cognitive domains, including memory, in patients with sporadic subcortical ischemic vascular dementia (SVaD). ${ }^{23)}$ Moreover, CADASIL and SVaD showed a similar pattern of cognitive deficit in a British CADASIL study. ${ }^{24)}$ Thus, these results support our hypothesis that the degree of CMBs correlates with memory dysfunction.

We also found strong associations of Scheltens scores with the executive functioning domain. It is generally thought that WMHs are associated with cognitive dysfunction. ${ }^{25)}$ Although Benisty et al. reported CADASIL patients with isolated WMHs present with executive and attention deficits, ${ }^{26)}$ the independent effects of WMH volume on cognition in CADASIL patients had not been described before. ${ }^{27)}$ Our results also demonstrated that three or more lacunes were an independent predictor of executive and attention domains. In our study, CADASIL patients with 2 or fewer lacunes did not show any cognitive dysfunction. We cannot exclude the possibility that one or 
two lacunes contributed to cognitive dysfunction in CADASIL. However, our results suggest that the impact of one or two lacunes on cognition is less significant than that of 3 or more lacunes. However, there are limitations in interpreting why there are other impacts on cognitive domains, depending on whether they are CMBs or lacunes. Analysis of the location of CMBs and lacunes has not been done in this study; it is difficult to make conclusions about the effect of CMBs on cognitive domains.

However, this study was a cross-sectional study. Thus, further prospective studies are needed to elucidate the association between CMBs and cognitive dysfunction. Another limitation of our study was that we were not able to investigate whether the location of CMBs is associated with cognition. However, recent studies of the relationship between the location of CMBs and cognition have produced conflicting results. ${ }^{28)} \mathrm{A}$ final limitation was that the severely disabled patients with CADASIL might have been excluded from our study.

We found that grade of CMBs is associated with memory dysfunction and that WMHs may contribute to the prediction of executive dysfunction, therefore, propose that CMBs and WMHs may be useful imaging markers to associated with cognitive dysfunction in CADASIL.

\section{CONFLICTS OF INTEREST}

The authors declare that they have no competing interests.

\section{ACKNOWLEDGEMENT}

The research was supported by the 2018 scientific promotion program funded by Jeju National University.

\section{REFERENCES}

1. Greenberg SM, Vernooij MW, Cordonnier C, Viswanathan A, AlShahi Salman R, Warach S, et al. Cerebral microbleeds: a guide to detection and interpretation. Lancet Neurol 2009;8:165-74.

2. Tatsumi S, Shinohara M, Yamamoto T. Direct comparison of histology of microbleeds with postmortem MR images: a case report. Cerebrovasc Dis (Basel, Switzerland) 2008;26:142-6.

3. Nandigam RN, Viswanathan A, Delgado P, Skehan ME, Smith EE, Rosand J, et al. MR imaging detection of cerebral microbleeds: effect of susceptibility-weighted imaging, section thickness, and field strength. AJNR Am J Neuroradiol 2009; 30:338-43.

4. Ayaz M, Boikov AS, Haacke EM, Kido DK, Kirsch WM. Imaging cerebral microbleeds using susceptibility weighted imaging: one step toward detecting vascular dementia. J Magn Reson Imaging 2010;31:142-8.

5. Pantoni L, Fierini F, Poggesi A. Thrombolysis in acute stroke patients with cerebral small vessel disease. Cerebrovasc Dis (Basel, Switzerland) 2014;37:5-13.

6. Thijs V, Lemmens R, Schoofs C, Görner A, Van Damme P, Schrooten M, et al. Microbleeds and the Risk of Recurrent Stroke. Stroke 2010;41:2005-9.

7. Joutel A, Corpechot C, Ducros A, Vahedi K, Chabriat H, Mouton $\mathrm{P}$, et al. Notch3 mutations in CADASIL, a hereditary adult-onset condition causing stroke and dementia. Nature 1996;383:707-10.

8. Lee JS, Choi JC, Kang SY, Kang JH, Lee SH, Kim JH, et al. Olfactory identification deficits in cerebral autosomal dominant arteriopathy with subcortical infarcts and leukoencephalopathy. Eur Neurol 2010;64:280-5.

9. Dichgans M. CADASIL: a monogenic condition causing stroke and subcortical vascular dementia. Cerebrovasc Dis (Basel, Switzerland) 2002;13 Suppl 2:37-41.

10. Choi JC, Kang S-Y, Kang J-H, Park J-K. Intracerebral hemorrhages in CADASIL. Neurology 2006;67:2042-4.

11. Lesnik Oberstein SA, van den Boom R, van Buchem MA, van Houwelingen HC, Bakker E, Vollebregt E, et al. Cerebral microbleeds in CADASIL. Neurology 2001;57:1066-70.

12. Liem MK, Lesnik Oberstein SA, Haan J, van der Neut IL, Ferrari MD, van Buchem MA, et al. MRI correlates of cognitive decline in CADASIL. A 7-year follow-up study 2009;72:143-8.

13. Lee JS, Kang C-H, Park SQ, Choi HA, Sim K-B. Clinical significance of cerebral microbleeds locations in CADASIL with R544C NOTCH3 mutation. PloS one 2015;10:e118163-e.

14. Kim HS, Lee DH, Ryu CW, Lee JH, Choi CG, Kim SJ, et al. Multiple cerebral microbleeds in hyperacute ischemic stroke: impact on prevalence and severity of early hemorrhagic transformation after thrombolytic treatment. AJNR Am J Roentgenol 2006; 186:1443-9.

15. Kim Y, Choi EJ, Choi CG, Kim G, Choi JH, Yoo HW, et al. Characteristics of CADASIL in Korea: a novel cysteine-sparing Notch3 mutation. Neurology 2006;66:1511-6.

16. Viswanathan A, Guichard JP, Gschwendtner A, Buffon F, Cumurcuic R, Boutron $\mathrm{C}$, et al. Blood pressure and haemoglobin A1c are associated with microhaemorrhage in CADASIL: a two-centre cohort study. Brain 2006;129:2375-83.

17. Dichgans M, Holtmannspotter M, Herzog J, Peters N, Bergmann M, Yousry TA. Cerebral microbleeds in CADASIL: a gradient-echo magnetic resonance imaging and autopsy study. Stroke 2002;33: 67-71.

18. Viswanathan A, Gschwendtner A, Guichard JP, Buffon F, Cumur- 
ciuc $\mathrm{R}$, O’Sullivan M, et al. Lacunar lesions are independently associated with disability and cognitive impairment in CADASIL. Neurology 2007;69:172-9.

19. Liem MK, van der Grond J, Haan J, van den Boom R, Ferrari MD, Knaap YM, Breuning MH, et al. Lacunar infarcts are the main correlate with cognitive dysfunction in CADASIL. Stroke 2007;38: 923-8.

20. Lee JS, Choi JC, Kang SY, Kang JH, Na HR, Park JK. Effects of lacunar infarctions on cognitive impairment in patients with cerebral autosomal-dominant arteriopathy with subcortical infarcts and leukoencephalopathy. Journal of clinical neurology (Seoul, Korea) 2011;7:210-4.

21. Epelbaum S, Benisty S, Reyes S, O’Sullivan M, Jouvent E, Düring $\mathrm{M}$, et al. Verbal memory impairment in subcortical ischemic vascular disease: a descriptive analysis in CADASIL. Neurobiol Aging 2011;32:2172-82.

22. Dichgans M, Markus HS, Salloway S, Verkkoniemi A, Moline M, Wang Q, et al. Donepezil in patients with subcortical vascular cognitive impairment: a randomised double-blind trial in CADA-
SIL. Lancet Neurol 2008;7:310-8.

23. Seo SW, Hwa Lee B, Kim EJ, Chin J, Sun Cho Y, Yoon U, et al. Clinical significance of microbleeds in subcortical vascular dementia. Stroke 2007;38:1949-51.

24. Charlton RA, Morris RG, Nitkunan A, Markus HS. The cognitive profiles of CADASIL and sporadic small vessel disease. Neurology 2006;66:1523-6.

25. Breteler MM, van Swieten JC, Bots ML, Grobbee DE, Claus JJ, van den Hout JH, et al. Cerebral white matter lesions, vascular risk factors, and cognitive function in a population-based study: the Rotterdam Study. Neurology 1994;44:1246-52.

26. Benisty S, Reyes S, Godin O, Hervé D, Zieren N, Jouvent E, et al. White-matter lesions without lacunar infarcts in CADASIL. J Alzheimers Dis 2012;29:903-11.

27. Dichgans M. Cognition in CADASIL. Stroke 2009;40:S45-S7.

28. van Es AC, van der Grond J, de Craen AJ, Westendorp RG, Bollen EL, Blauw GJ, et al. Cerebral microbleeds and cognitive functioning in the PROSPER study. Neurology 2011;77:1446-52. 